\title{
Violencia contextual, ciberacoso y sintomatología de estrés postraumático en universitarios de Ciudad Juárez, Chihuahua
}

\section{Contextual violence, cyberbullying, and posttraumatic stress symptomatology in university students in Ciudad Juárez, Chihuahua}

María Nieves González Valles

Alberto Castro Valles

María Elena Vidaña Gaytán

Universidad Autónoma de Ciudad Juárez

\author{
Georgina Lozano Razo
}

Universidad Nacional Autónoma de México

\section{Resumen}

En el contexto fronterizo del norte de México, signado en los últimos años por niveles de violencia social sin precedente, se analizó (como objetivo del estudio) la relación de la frecuencia a la exposición a la violencia comunitaria o contextual con la violencia ejercida a través de dispositivos electrónicos y los síntomas de estrés postraumático en jóvenes estudiantes universitarios residentes de Ciudad Juárez. Mediante un estudio cuantitativo correlacional con temporalidad transversal, se aplicaron tres instrumentos de autorreporte con escalamiento Likert a 300 estudiantes universitarios donde se identificaron las relaciones significativas entre las experiencias de exposición a la violencia comunitaria o contextual, el ciberacoso y la sintomatología de estrés postraumático. Las escalas resultaron con fiabilidad aceptable (con un alpha mayor $\mathrm{a}=.748$ ). Veintidós por ciento de los estudiantes que percibieron al menos un acto de violencia hacia sí mismos, hacia familiares o hacia amistades cercanas y/o en línea presentaron más de dos síntomas de EPT. A través de la prueba estadística de Pearson en el programa SPSS, se encontraron correlaciones positivas estadísticamente significativas entre la exposición a violencia contextual en escuelas, colonias y lugares de diversión con las acciones de violencia en línea de ciberacoso. Asimismo, se encontraron correlaciones significativas entre la violencia directa e indirecta con sintomatología de estrés postraumático, principalmente de tipo presencial en escuelas y en centros de diversión.

Palabras clave: violencia contextual, violencia social, ciberacoso, estrés postraumático, jóvenes.

Nota del autor

María Nieves González Valles, Facultad de Psicología, Universidad Autónoma de Ciudad Juárez. Alberto Castro Valles, Facultad de Psicología, Universidad Autónoma de Ciudad Juárez. María Elena Vidaña Gaytán, Facultad de Psicología, Universidad Autónoma de Ciudad Juárez. Georgina Lozano Razo, Facultad de Psicología, Universidad Nacional Autónoma de México.

La correspondencia en relación con este artículo debe dirigirse María Nieves González Valles, Universidad Autónoma de Ciudad Juárez, Av. Plutarco Elías Calles \#1210, Fovissste Chamizal, C.P. 32310, Ciudad Juárez, Chihuahua, México. Correo electrónico: mgonzale@uacj.mx 


\begin{abstract}
In recent years, Mexico's northern border has seen unprecedented levels of social violence. With this as the backdrop of our research study, the objective was to analyze the relationship between the frequency of exposure to community or contextual violence and violence through electronic means, and some indicators of posttraumatic stress in young university students from Ciudad Juárez. Through a cross-sectional, quantitative, correlational study, three self-report instruments were applied to 300 university students. Significant relationships between experiences of exposure to community or contextual violence, cyberbullying, and post-traumatic stress symptomatology were identified. The scales were found to be reliable (with an alpha greater than $\mathrm{a}=.748$ ). Twenty-two percent of students who reported at least one act of violence against themselves, against family members or close friends, and/or online, also reported experiencing two or more indicators of posttraumatic stress. Statistically significant positive correlations were found between exposure to contextual violence in schools, neighborhoods, and places of leisure, and cyberbullying. Similarly, there were significant correlations between direct and indirect violence and posttraumatic stress symptomatology, primarily in brickand-mortar schools and entertainment centers.
\end{abstract}

Keywords: contextual violence, social violence, cyberbullying, posttraumatic stress, youth.

\section{Introducción}

Declarada desde 1966 como un problema de salud pública, la violencia se manifiesta multidimensionalmente y se explica a partir de la confluencia de factores sociales, económicos y culturales, considerando otros de índole contextual, institucional e individual (Wieviorka, 1997). La Organización Mundial de la Salud (OMS) definió a la violencia como "el uso deliberado de la fuerza física o el poder ya sea en grado de amenaza o efectivo, contra uno mismo, otra persona o un grupo o comunidad, que cause o tenga muchas probabilidades de causar lesiones, muerte, daños psicológicos, trastornos del desarrollo o privaciones" (2002, p.5). En la sociedad actual se pueden distinguir tres grandes tipos de violencia: (a) autoinflingida, abarca cualquier conducta intencional y dañina dirigida hacia sí mismo; (b) interpersonal o conductas violentas entre individuos, donde una primera modalidad incluye a la violencia intrafamiliar o de pareja, y en una segunda, se consideran la violencia comunitaria, juvenil, sexual y otras según contextos determinados como la violencia escolar y laboral; (c) colectiva, aquella motivada por fines políticos, sociales o económicos de un grupo social que incluye conflictos armados, genocidio y crimen organizado (p.6).

América Latina se ha identificado como la región más violenta del mundo en los últimos años. La mayoría de los países en este continente ha experimentado modalidades de violencia que, en las últimas décadas, se configuran con rostros de violencia social generada por 
grupos paramilitares o diversas organizaciones criminales que afectan a la población (OMS, 2002). La tasa mundial de homicidios en 2002 estimaba 8.8 por cada 100000 habitantes. En el 2008, y de acuerdo con Briceño-León (2008, p.108), los países con niveles de violencia alta, como es el caso de México y Brasil, cuentan con una tasa de homicidios entre 17.6 y 26.4.

Alvarado (2013) señaló que, hasta 2007, las tasas de homicidio en algunos países latinoamericanos presentaron descensos. Sin embargo, el fenómeno aumentó en México de manera considerable a partir de 2008, pasando de nueve a más de 21 homicidios por cada 100000 habitantes. Cifras del Banco Mundial estiman que, en la primera década del siglo XXI, alrededor de 139000 mexicanos fueron víctimas de homicidio. Si bien de 2000 a 2007 la tasa había descendido de 11.0 a 8.4 por cada 100000 habitantes, a partir de 2008 la cifra no solo se recuperó, sino se multiplicó alcanzando los 28.0 en 2010 (BM, 2012). Se calcula que tan solo en el periodo 2007 a 2012 se produjeron más de 120000 muertes producto de la violencia extrema en México. En la última década, esta cifra se incrementó, pues hasta el 2016 se han identificado más de 300000 homicidios (Rodríguez-Ferreira, 2016).

En este entramado, Ciudad Juárez ha sido epicentro de modalidades de violencia social sin precedente, donde a la impunidad ante los feminicidios mundialmente conocidos —ante un escenario de precariedad laboral - se suma la disputa del territorio por bandas del crimen organizado especializados no solo en la venta de drogas ilegales, sino también en secuestro, homicidios a gran escala y todas las modalidades de robo y extorsión. En palabras de ArteagaBotello (2003), "la confluencia de factores sociales, culturales e individuales dibuja de manera nítida el espacio de la violencia en esta urbe fronteriza" (p.119). La escalada en los niveles de violencia transformó a un país proveedor de drogas en uno con verdaderas zonas de guerra (Rodríguez-Ferreira, 2016). La respuesta del Estado ante dicha problemática vino a agravar las condiciones de vida de los juarenses, pues la presencia de las fuerzas armadas de la policía y el ejército aumentó los niveles de miedo, inseguridad, impotencia e indignación de los ciudadanos (Alvarado, 2013).

En el transcurso de los siguientes años, la sociedad continúa atestiguando el ejercicio sistemático de la violencia donde las tasas de homicidios disminuyeron para luego volver a incrementar (Observatorio Nacional Ciudadano, 2015). Los costos indirectos del crimen organizado se presentan multidimensionalmente, a nivel laboral, familiar, social e individual, en estos escenarios se suele experimentar sufrimiento, miedo y pérdida en la calidad de vida, entre otros. Estos costos finalmente impiden desarrollar el potencial productivo a los seres humanos (Jaitman, 2015).

Independientemente de los esfuerzos implementados para disminuir, atender o prevenir la escalada de violencia colectiva, diversos estudios insisten en que esta 
problemática debe ser abordada como un problema de salud pública (Krug et al., 2002). Dado que los jóvenes representan un porcentaje muy importante en términos poblacionales $\mathrm{y}$, al mismo tiempo, resultan más vulnerables ante distintas problemáticas, en el presente estudio nos enfocamos en conocer la relación de la exposición a la violencia comunitaria y mediante redes electrónicas a este grupo etario con sintomatología de estrés postraumático.

Alvarado (2013) se propuso conocer la percepción, las experiencias y reacciones de los jóvenes ante la violencia en América Latina. Específicamente, propuso conocer sus vivencias, experiencias de agresiones, lesiones en su contexto cotidiano y cómo esto afecta su vida. En el estudio de Alvarado participaron jóvenes habitantes de localidades en Argentina, Brasil, Colombia, Guatemala, y otros de varias ciudades de México - específicamente Jalapa, Ciudad Juárez, León y Ciudad de México Los hallazgos de los cinco países coinciden en que los participantes comparten la percepción de un hábitat con barrios seguros que al mismo tiempo resultan peligrosos por los altos índices de delitos que se presentan, como lo son la venta y uso de drogas ilegales y la portación y venta de armas de fuego. La explicación delineada por el autor en torno a este fenómeno alude a un posible proceso de adaptación a la violencia de los entornos de este grupo etario (p.238).

Por su parte, Chávez y colaboradores (2015) encontraron que las emociones más duraderas asociadas a la violencia psicosocial en los adultos coahuilenses jóvenes son distintas por sexo. La mayoría de las emociones negativas asociadas se presentan con mayor frecuencia en las mujeres. Como ejemplo, el miedo tiene mayor recurrencia en el $69.2 \%$ de las mujeres y en el $30.8 \%$ de los varones; la ansiedad se experimenta en $66.7 \%$ y $33.3 \%$; la tristeza 61.1 $\%$ y $38.9 \%$ respectivamente; sin embargo, el enojo se constituye la emoción más duradera en los varones con un $56.3 \%$ contra $43.8 \%$ (p.17).

Asimismo, Laca-Arocena y NavarroCamarena (2013) evaluaron las actitudes (es decir, cómo responden afectiva, cognitiva $\mathrm{y}$ conductualmente los jóvenes colimenses ante la violencia social) y lo relacionaron con el bienestar subjetivo. En sus resultados reportan efectos negativos por la percepción de la violencia, así como un fenómeno paradójico donde los jóvenes refieren una elevada alarma ante el alta en las tasas de violencia social, pero que es acompañada de reacciones emocionales no acordes con los eventos vividos y, por tanto, no modifican sus comportamientos. Por lo que, a decir de los investigadores, resulta incongruente la dimensión cognitiva de la actitud con la afectiva y la comportamental, pues los niveles de satisfacción con la vida y bienestar social se presentan en rangos elevados, lo que se ha considerado característico de la cultura mexicana.

Al hurgar en los efectos de la violencia colectiva en otros grupos poblacionales, Leiner et al. (2015) buscaron comparar trastornos emocionales y comportamentales 
en niños expuestos a la violencia colectiva en comunidades de ambos lados de la frontera México-Estados Unidos. De su análisis concluyen que la victimización producto del crimen se erige como el principal problema de salud pública, al aumentar los riesgos de padecer desórdenes psiquiátricos y psicológicos en la infancia. Algunos de estos desórdenes pueden ser estrés postraumático, abuso de sustancias, problemas de salud. Como consecuencia de ello, posiblemente se proyecten muchos de esos niños como futuros perpetradores de distintas modalidades de violencia.

Bajo la perspectiva de la violencia y sus costos como un problema de salud pública, resulta relevante analizar otras experiencias de violencia colectiva en otros países, puesto que tienen sus propias particularidades. Comparten con México la presencia de grupos de crimen organizado, tráfico de drogas y homicidios a gran escala. El caso colombiano, por ejemplo, brinda referentes importantes: un grupo de investigadores en ese país analizó datos de 5 064 adultos que recibieron atención clínica por parte de Médicos Sin Fronteras (MSF) entre 2005 y 2009. MSF integra el trabajo psicológico y psicosocial a sus actividades de salud primaria en Colombia (MSF, 2010). Muchos de los usuarios atendidos por MSF han descrito haber sido víctimas de actos de violencia directa como amenazas, palizas, violaciones, restricciones de movilidad o desplazamientos. Es vivida como el daño corporal, físico y/o mental comprobable y, en principio, evidente (MSF, 2013).
A partir de los resultados, los autores concluyeron que la violencia directa, ligada al conflicto armado, afecta y se manifiesta como daño o alteración de la salud mental, como estrés postraumático, depresión mayor y ansiedad, problemas de adaptación y un mayor grado de exposición a otros tipos de violencia, como la intrafamiliar, sexual o social. Asimismo, diagnosticaron el trastorno adaptativo (18.6\%), problemas de relación y problemas asociados con el abuso o la negligencia (17.5\%), depresión mayor (11.3\%), duelo (8.9\%), y los trastornos del estado de ánimo (8.2 \%). Por la propia naturaleza de tales diagnósticos principales, se asume que están vinculados a circunstancias vitales externas devastadoras, que se consideran como normales ante circunstancias anormales o extremas (MSF, 2010).

Años más tarde, el mismo grupo MSF reporta la atención a 4455 pacientes durante 2012, quienes fueron afectados por enfrentamiento, lo que pone en evidencia las secuelas que la lucha armada deja en la salud mental. Gran parte de las personas que han acudido a la consulta clínica de MSF ha vivido uno o más hechos relacionados con la violencia $(67 \%)$ y con la separación o pérdida (14\%). Como consecuencia de ello y de otros factores de riesgo, se detectan síntomas relacionados con depresión y ansiedad $\mathrm{o}$ síntomas somáticos de origen psicológico. Para cada paciente, los psicólogos podían registrar hasta tres síntomas individuales. Así, humor triste, preocupación constante y ansiedad/estrés tuvieron las frecuencias más altas, estando 
presentes en el $38 \%, 34 \%$ y $29 \%$ de los pacientes, respectivamente (MSF, 2013).

A modo de síntesis, en la historia reciente de varios países latinoamericanos han quedado grabados periodos de violencia extrema sobre sus poblaciones. Estos han debilitado el tejido social y aumentado exponencialmente los trastornos psicológicos y psiquiátricos (Andrade-Salazar, 2008). De modo que el atestiguamiento de olas de crímenes y violación masiva de derechos humanos con frecuencia genera problemas de estrés postraumático.

Ubicados en otro nivel, en el análisis de las modalidades de violencia comunes entre estudiantes, en los primeros años de las manifestaciones de la modalidad de violencia interpersonal, se asumía que el ciberacoso o cyberbullying era propio de los adolescentes; a causa de esto, se enfocaba el análisis del fenómeno en este grupo etario. Por ello, Buelga et al. (2010) analizaron la prevalencia de victimización entre adolescentes a través de dispositivos móviles e internet; observaron que el fenómeno padecido se da con mayor frecuencia en los primeros años de la secundaria, donde el $24.6 \%$ ha sido acosado a través del teléfono móvil en el último año, mientras el 29 $\%$ ha sido victimizado a través de la internet. Otras investigaciones también en adolescentes reportan que el $30.1 \%$ ha sufrido algún tipo de ciberagresión con mayor prevalencia en mujeres, donde las víctimas tienen menor autoestima con mayor sintomatología depresiva, cogniciones de maltrato y exposición a la violencia. Sin embargo, la investigación pone de manifiesto que el $22.8 \%$ de la muestra comparte la condición de víctima y agresor de acuerdo con lo encontrado por Estévez et al. (2010).

En México, Emir (2015) reportó que el 12 $\%$ de los adolescentes entre los 12 y 17 años son víctimas de ciberbullying, de acuerdo con los hallazgos de académicos investigadores de la Universidad Nacional Autónoma de México. El autor indicó que las víctimas son aquellas percibidas como débiles y que son señaladas por sus diferencias individuales como etnia, padecimiento de alguna discapacidad, condición socioeconómica, rasgos de personalidad como el retraimiento o introversión, por destacar académicamente o con problemas escolares, por su condición física de altura o complexión y preferencias sexuales, entre otras. También, García-Maldonado et al. (2012) agregaron que las víctimas de ciberbullying mantienen un uso prolongado de internet en redes sociales y estimaron una posible relación con baja autoestima, insomnio, enuresis, ansiedad, cefalea, dolor abdominal, alteraciones emocionales $\mathrm{y}$ dificultades académicas, que de no atenderse adecuadamente puede generar sintomatología psicopatológica severa. Por su parte, VegaLópez et al. (2013) señalaron que en México la prevalencia del ciberacoso en adolescentes se estima en $14.13 \%$, principalmente a través de la transmisión de texto o imágenes insultantes por los teléfonos móviles.

Otras investigaciones sobre el ciberacoso se han enfocado en población universitaria. Serrano 
Barquín y Serrano Barquín (2014) elaboraron un estudio para conocer la opinión de los estudiantes universitarios sobre el ciberacoso. Desde una perspectiva cualitativa, entrevistaron a estudiantes quienes expresaron que las burlas son un comportamiento habitual entre los estudiantes universitarios, incluso propio de la cultura mexicana, normalizado y que, por tanto, no se puede considerar un problema grave.

Asimismo, Prieto-Quezada et al. (2015) condujeron una investigación en estudiantes universitarios de Guadalajara México. Con una muestra representativa, reportan la existencia de esta modalidad de acoso escolar también en niveles de educación superior entre un $20 \%$ y $40 \%$ de los estudiantes, en las modalidades de insultos y palabras ofensivas que llevan a la ridiculización, mientras otra proporción reporta acoso sexual, y otros más el robo de contraseñas. De ello se asume que los universitarios puedan estar experimentando sentimientos asociados como el miedo, desconfianza y es posible que ciertos niveles de paranoia ante la vulnerabilidad del robo de identidad y datos personales. De acuerdo con De la Rosa y Cárdenas (2012), los efectos mostrados en síntomas de TEPT no solo están relacionados con experiencias de violencia vivida sino también con experiencias de violencia social en contextos escolares, habitacionales y en lugares de diversión, así como en el ambiente e internet.

El DSM-IV consideraba que el TEPT se genera cuando la persona ha sufrido o ha presenciado una agresión física o amenaza que pone en juego la propia vida o la de alguien más, y cuando la reacción emocional que se experimenta implica una respuesta intensa de miedo, horror o indefensión (Echeburúa et al., 1997). Por su parte, Carvajal (2002) enfatizaba que el TEPT se caracteriza por una triada sintomática de fenómenos invasores, síntomas de hiperalerta y conductas de evitación. Según la Asociación Americana de Psiquiatría (APA), la característica esencial del TEPT es el desarrollo de síntomas específicos tras la exposición a uno o más eventos traumáticos.

Partiendo del Manual de Diagnóstico para las Enfermedades Mentales (DSM) quinta versión, existen tres factores esenciales del cuadro clínico de esta reacción al estrés: (a) la reexperimentación de la experiencia vivida en forma de pesadillas o de imágenes constantes e involuntarias; (b) las conductas de evitación de situaciones asociadas al hecho traumático, y (c) las respuestas de hiperactivación, como dificultad para concentrarse, irritabilidad, problemas para conciliar el sueño y respuestas exageradas de sobresalto (APA, 2014). Ante un acontecimiento traumático, se genera una demanda del organismo donde a menudo los mecanismos de adaptación resultan insuficientes para adaptarse a la situación estresante. Entre los principales estresores potenciales para la aparición del TEPT, se reconocen la participación en situaciones de guerra, sea como combatiente, pero también como población civil; ser víctima de violencia interpersonal; el haber sido víctima de delitos de alto impacto como el secuestro o 
la tortura, entre otros ya referidos en la anterior versión del DSM-IV (APA, 1995).

Andrade-Salazar (2008) realizó un estudio con 36 familias víctimas del desplazamiento en Colombia, específicamente con padres y madres con edades de 20 a 40 años, buscando tipificar las patologías en salud mental derivadas del conflicto armado en ese país. Entre las medidas utilizadas para alcanzar su objetivo, a los participantes les fue aplicada la escala de trauma de Davidson para tipificar los trastornos de estrés postraumático. En sus hallazgos, el autor reporta que el $100 \%$ de la población presentó indicadores del citado trastorno, con una gravedad extrema del 50\%, y moderada del $40 \%$ (p.111), con presencia de somatizaciones, recuerdos recurrentes, anhedonia, episodios de pánico, reviviscencia de los acontecimientos, evitación de conversaciones y lugares referidos a los sucesos. En el 50\% dicha sintomatología apareció diariamente interfiriendo en la vida cotidiana de los participantes en los ámbitos psicosociales, afectivos y laborales, mientras en el $20 \%$ su frecuencia fue de 2 o 3 veces en el lapso de una semana. Esto a menudo genera altos montos de ira incontrolable que se traduce en comportamientos agresivos hacia la propia familia (p.123).

En el caso de Perú, también en las últimas dos décadas del siglo pasado sus habitantes presenciaron una de las etapas de graves conflictos armados, como resultado de la violencia política infligida a su población. En ese contexto, Herrera-López y Cruzado
(2014) se propusieron conocer la prevalencia del trastorno de estrés postraumático, la comorbilidad psiquiátrica asociada en los habitantes de una comunidad campesina. Sus resultados señalaron la experiencia de 2.26 eventos traumáticos en promedio, mientras la prevalencia del TEPT se dio en el $25.6 \%$ de los participantes. Es importante aclarar que, sin excepción, los habitantes de esta comunidad rural experimentaron al menos un trastorno psiquiátrico o psicológico, específicamente mayores niveles de ansiedad generalizada y depresión.

En estudiantes universitarios se ha identificado rumiación deliberada con reevaluación cognitiva positiva con estrategias de afrontamiento de apoyo social y moralreligioso, pero con baja expresividad emocional relacionada el crecimiento postraumático (Cova et al., 2011). Asimismo, en otro estudio donde la población juvenil estudiantil universitaria del Estado de México estuvo expuesta durante su infancia y adolescencia, en su mayoría, a actos de violencia en sus distintas manifestaciones percibiéndolos amenazantes para la integridad y continuidad de su vida, se identificó un $78 \%$ de prevalencia de sucesos traumáticos (Mendoza et al., 2013).

Por su propia condición de jóvenes que nacieron a la par de una revolución tecnológica que amplía los canales de comunicación exponencialmente, de manera privilegiada, este grupo poblacional queda a un mismo tiempo expuesto a otras modalidades de 
violencia ejercida precisamente a través de las plataformas sociales y medios de comunicación electrónicos. Por lo cual anterior, en lo que sigue nos trasladamos a otro ámbito de análisis: el ciberacoso o cyberbullying. Se trata de una nueva modalidad de intimidación y acoso escolar ejercida por un individuo o grupos durante un periodo de tiempo por medio del uso de dispositivos electrónicos.

A diferencia de otras formas de acoso escolar, en este caso al agresor le resulta fácil quedar en el anonimato, por lo que la conducta agresiva e intencional suele repetirse en el tiempo aumentando los sentimientos de indefensión de la víctima. Las modalidades de violencia a través de las nuevas tecnologías de la información y comunicación, pueden ser hostigamiento, persecución, denigración, violación de la intimidad, exclusión social y, hasta suplantación de identidad (Willard, 2006 y 2007, en Buelga et al., p.784). En contextos de violencia masiva social continua y permanente, cada vez más se reconoce que esta forma parte de la vida cotidiana, siendo normalizada por niños y jóvenes, puesto que es presenciada o experimentada permanentemente de manera directa o a través de los medios de comunicación (González-Valles \& Romero-Cruz, 2015).

Finalmente, en este análisis es importante retomar la discusión en torno a algunas explicaciones relativas a la aparente indiferencia o falta de correspondencia entre los eventos vividos y las reacciones presentadas en algunos jóvenes (Laca-Arocena y Navarro-Camarena,
2013). En este sentido, Abramovay (2005) refiere la emergencia de una posible normalización de la violencia, sobre todo en determinados ámbitos, como el escolar, donde el fenómeno resulta preocupante y sus orígenes no resultan identificables a primera vista, sino más bien, pareciera tratarse de una violencia gratuita, cada vez más naturalizada. Así, el estudio se guio por la interrogante: ¿existe relación significativa entre la frecuencia de experiencias de violencia comunitaria o contextual y del ciberacoso con síntomas del estrés postraumático en estudiantes universitarios de Ciudad Juárez, Chihuahua?

\section{Método}

El objetivo de la investigación fue conocer la relación entre la frecuencia de experiencias de violencia comunitaria o contextual y del ciberacoso con síntomas del estrés postraumático en estudiantes universitarios de Ciudad Juárez, Chihuahua. Esto permite, en un enfoque cuantitativo con temporalidad transversal, visibilizar los efectos psicosociales en los jóvenes que han experimentado o presenciado una actividad violenta en su vida cotidiana. Se identifica la cantidad de experiencias vividas presenciando de manera directa o indirecta hechos de daño intencional físico o psicológico en el contexto en que se desenvuelve cotidianamente, categorizadas como violencia contextual, y en ambientes de interacción en red social mediante plataformas digitales, categorizadas como ciberacoso. El estrés postraumático es una afectación en el estado 
biológico, psicológico, emocional y social, ocasionada por la experiencia de haber vivido un evento ambiental sorpresivo interpretado como riesgo para la vida o la integridad personal, caracterizado por dificultades en la memoria, pensamiento regresivo, ansiedad y angustia, entre otros. Por lo anterior, se considera como planteamiento hipotético la existencia de una relación significativa entre la frecuencia de las experiencias de violencia contextual y de ciberacoso con la presencia de síntomas de estrés postraumático.

\section{Participantes}

Se describieron las relaciones de las variables de estudio con una muestra de estudiantes universitarios no probabilística por sujetos voluntarios a conveniencia. Se tamizaron 786 estudiantes cuyas preguntas refirieron a contestar categóricamente (sí/no) si habían experimentado alguna experiencia de violencia comunitaria, de ciberacoso y si tenían al menos dos de los síntomas de estrés postraumático. La muestra objetivo fue de 300 (38\%) de los estudiantes tamizados quienes contestaron los instrumentos y una sección de datos demográficos. $51 \%$ fueron mujeres y $49 \%$ hombres, con media de edad e 22 años (DS=2.5) entre 18 y 33 años. El $85 \%$ de la muestra eran originarios de Ciudad Juárez, Chihuahua. 86.3\% reportaron ser solteros; 9\%, casados, y $4.7 \%$ en unión libre. El $50.3 \%$ trabaja y el $49.7 \%$ no trabaja; el $75 \%$ reportó practicar deporte. El 50 $\%$ asiste al turno matutino, $28 \%$ al vespertino y el $22 \%$ asiste en turno mixto. $44 \%$ cursan semestres de nivel avanzado ( $7 .^{\circ}$ semestre o más); $39 \%$, intermedio ( $4 .^{\circ}-6 .^{\circ}$ semestre), y el $17 \%$, principiante (1.er- 3.er semestre), con un promedio de calificaciones de $\mathrm{x}=8.8(\mathrm{DS}=.55)$ entre 7.0 y 10.0 .

\section{Procedimiento}

En una primera etapa se seleccionaron los instrumentos de la escala de victimización (Ruíz, 2007), la escala de exposición de la violencia elaborada por Gurrola-Peña et al (inédito), la escala breve de ciberacoso (Estévez et al., 2010) y la escala breve de estrés postraumático (Breslau et al, 1999). Asimismo, se construyó un breve cuestionario de tamizaje para ser aplicado en una universidad pública de Ciudad Juárez, Chihuahua, donde se obtuvieron los permisos correspondientes de administradores educativos. Se tamizaron 786 estudiantes en diversos espacios como aulas, biblioteca y espacios abiertos de cuatro campus universitarios de la máxima casa de estudios. En una segunda etapa, se analizó el tamizaje y se logró contactar a 300 estudiantes universitarios; estos contestaron los instrumentos seleccionados en un espacio áulico controlado con duración de 45 minutos aproximadamente bajo consentimiento informado: se aseguró la confidencialidad y anonimato de los participantes. Finalmente, en una tercera etapa, se realizaron análisis estadísticos descriptivos, diferenciales por sexo y de correlación bivariada de Pearson en el programa estadístico para las ciencias sociales (SPSS) versión 22. 


\section{Instrumentos}

Los instrumentos utilizados en la recolección de datos fueron la escala de victimización elaborada por Ruíz (2007) con alpha de Cronbach de $\mathrm{a}=.898$ en 15 ítems que refieren haber vivido experiencias de violencia, con puntuaciones de 0 a 15 para victimización directa y puntuaciones de 0 a 17 para victimización indirecta. Además, se empleó un cuestionario denominado escala de exposición de la violencia (EVV) elaborado por Gurrola-Peña et al (inédito) de la Universidad Autónoma del Estado de México, compuesto por 36 reactivos de tipo Likert con sentido de frecuencia del 1 al 5 , con y un alpha total $\mathrm{a}=.94$ en cinco factores $(49.75 \%$ de varianza explicada): victimización contextual no presencial $(a=.90$, 8 ítems), victimización contextual presencial ( $\mathrm{a}=85,7$ ítems), victimización contextual en la colonia ( $\mathrm{a}=.88,8$ ítems), victimización contextual en los lugares de diversión $(\mathrm{a}=.86$, 6 ítems) y victimización en la escuela $(\mathrm{a}=.80,6$ ítems).

Asimismo, la escala breve de cyberbullying (CBQ-V) es un cuestionario de victimización que incluye once modalidades de cyberbullying elaborado por Estévez et al. (2010). Este instrumento, compuesto por 11 reactivos tipo Likert en sentido de frecuencia: nunca (1) algunas veces (2) y con frecuencia (3), identifica acciones sufridas de violencia mediante el uso de internet y de teléfonos móviles, smartphone, etc., ha mostrado propiedades psicométricas excelentes en relación con la estructura factorial y la consistencia interna de alpha $\mathrm{a}=.95$; además permite estimar tasas de prevalencia (p.82).

Por último, la escala breve de estrés postraumático (PTSD, Short Screening Scale for DSM-IV Posttraumatic Stress Disorder), consta de siete reactivos con punto de corte de 4; posee $80 \%$ de sensibilidad, $97 \%$ de especificidad, valores positivos $71 \%$, y $98 \%$ de valores predictivos negativos con regresión lineal binaria. Con una muestra de 2181 sujetos con edades de 18 a 45 años que habían experimentado exposición a eventos traumáticos, tomando como base el DSM-IV, Breslau, Peterson, Kessler y Shultz (1999) elaboraron este instrumento de tamizaje para identificar sintomatología de estrés postraumático en estadounidenses con alpha de $\mathrm{a}=.987$; representó un método eficiente para identificar dicha sintomatología, dada la limitación de recursos. Los autores presentan esta versión corta de tamizaje integrada por siete síntomas contrastados mediante la entrevista diagnóstica del Instituto Nacional de Salud Mental en los Estados Unidos.

\section{Consideraciones éticas}

Se incluyó un consentimiento informado, donde se aseguró la confiabilidad de los datos y el anonimato, así como la libre participación para responder cinco preguntas tipo screening de tamizaje y posteriormente participar contestando los instrumentos utilizados de acuerdo con los lineamientos establecidos por el código ético del psicólogo (SMP, 2009). 


\section{Resultados}

En la muestra evaluada de 300 estudiantes universitarios, la Escala de Victimización (Ruíz, 2009) obtuvo un alpha de Cronbach de $a=.806$ en 15 elementos, por lo que fue consistente y con apreciación confiable. Se observó que el $30 \%$ (90) experimentaron robos con violencia, $23 \%$ (68) presenciaron violencia en conocidos, $11 \%$ (33) extorsión económica, 10\% (30) agresión sexual, 8\% (25) persecución por desconocidos, 8\% (23) agresión o amenazas físicas, $6 \%$ (20) secuestro con agresión sexual y $4 \%$ (11) presenciaron muerte violenta de una persona cercana (Tabla 1). La victimización directa - haber sufrido violencia- se observó mayor en las mujeres que en los hombres $(\mathrm{Mx}=3.9, \mathrm{Hx}=3.6)$ sin diferencia significativa, pero la indirecta - haber presenciado violencia en otros - fue también mayor en mujeres que en los hombres $(\mathrm{Mx}=7.6, \mathrm{Hx}=6.5)$ con diferencia significativa $(\mathrm{F}=4.51$, sig.=.034).

Tabla 1

Experiencia reportada de violencia directa por sexo

\begin{tabular}{lllll}
\hline \multirow{2}{*}{ Experiencia de violencia } & \multicolumn{2}{c}{ Mujeres } & \multicolumn{3}{c}{ Hombres } \\
& Recuento & Porcentaje & Recuento & Porcentaje \\
\hline Robos con violencia & 41 & $46 \%$ & 49 & $54 \%$ \\
Violencia en conocidos & 28 & $41 \%$ & 40 & $59 \%$ \\
Extorsión económica & 16 & $49 \%$ & 17 & $51 \%$ \\
Agresión sexual & 19 & $64 \%$ & 11 & $36 \%$ \\
Persecución por desconocidos & 17 & $68 \%$ & 8 & $32 \%$ \\
Agresión o amenazas físicas & 9 & $40 \%$ & 14 & $60 \%$ \\
Secuestro y agresión sexual & 12 & $60 \%$ & 8 & $40 \%$ \\
Muerte violenta de una persona cercana & 7 & $63 \%$ & 4 & $37 \%$ \\
Total & 149 & $51 \%$ & 151 & $49 \%$ \\
\hline
\end{tabular}

En la escala de exposición a la violencia (EEV) (Gurrola, et al. s/a), obtuvo un alfa de Cronbach de $a=.960$ con 35 elementos. La consistencia interna por factor resultó en factor $1(\mathrm{a}=.926,8)$, factor $2(\mathrm{a}=.911,7)$, factor $3(a=912,8)$, factor $4(a=911,6), y$ factor $5(a=843,5)$. El $30 \%$ de los participantes reportaron violencia contextual no presencial de al menos tres hechos de violencia a través de amigos, familiares y conocidos; $18 \%$, violencia contextual presencial en un promedio de dos eventos; 38\% del total de la muestra reportó exposición de violencia contextual presencial con cinco eventos promedio de violencia en 
su colonia o sección de residencia; $45 \%$ refirió al menos dos eventos violentos en lugares de diversión y $20 \%$ en espacios escolares con dos eventos promedio. Los hombres universitarios puntuaron mayor $(\mathrm{Hx}=14.6)$ que las mujeres $(\mathrm{Mx}=13.6)$ en victimización no presencial, presencial $(\mathrm{Hx}=10.3, \mathrm{Mx}=9.7)$ y lugares de diversión $(\mathrm{Hx}=11.9, \mathrm{Mx}=11.6)$, mientras que las mujeres tuvieron mayores puntuaciones en victimización en la colonia $(\mathrm{Mx}=18.3, \mathrm{Hx}=16.2)$ y en la escuela $(\mathrm{Mx}=7.8, \mathrm{Hx}=7.7)$. Sin embargo, la diferencia por sexo solo fue significativa en la colonia $(\mathrm{F}=6.014$, sig. $=.015)$.

La escala breve de cyberbullying CBQ-V (Estévez et al., 2010) obtuvo un alfa de $\mathrm{a}=.802$ con 11 elementos. Según la Tabla 2, el $49 \%$ ha tenido al menos una experiencia de ciberacoso, el cual correspondió al haber vivido violencia contextual presencial. No hubo diferencia significativa por sexo, aunque la tendencia fue mayor en varones $(\mathrm{x}=1.01)$ que en las mujeres $(\mathrm{Mx}=.97)$.

Tabla 2

Frecuencia de experiencia de ciberacoso

\begin{tabular}{lllll}
\hline \multirow{2}{*}{ İtems-ciberacoso / n=300 } & \multicolumn{2}{c}{ Algunas veces } & \multicolumn{2}{c}{ Con frecuencia } \\
& Recuento & Porcentaje & Recuento & Porcentaje \\
\hline Recibir mensajes de mail amenazantes o insultantes & 43 & $14 \%$ & $5(2)$ & $2 \%$ \\
Recibir mensajes de celular amenazantes o insultantes & 48 & $16 \%$ & $5(2)$ & $2 \%$ \\
Subir imágenes mías humillantes en internet & 17 & $6 \%$ & $/$ & $/$ \\
Subir comentarios negativos sobre mí en internet. & 35 & $12 \%$ & $4(1)$ & $1 \%$ \\
Usurpar mi identidad & 37 & $12 \%$ & $3(1)$ & $1 \%$ \\
Grabarme en video o fotografiarme mientras me obligan a & 8 & $3 \%$ & $1(.3)$ & $0.3 \%$ \\
hacer algo humillante & & & & \\
Grabarme en video o fotografiarme mientras me agreden & 6 & $2 \%$ & $/$ & $/$ \\
físicamente & 18 & $6 \%$ & $1(.3)$ & $0.3 \%$ \\
Difundir información confidencial mía en línea & 26 & $9 \%$ & $1(.3)$ & $0.3 \%$ \\
Aislarme en las redes sociales & 10 & $10 \%$ & $1(.3)$ & $0.3 \%$ \\
Recibir masivamente mensajes muy amenazantes. & & $2 \%$ & $/$ & $/$ \\
Grabarme realizando conductas sexuales y difundirlas en & 7 & & & \\
línea & & & & \\
\hline
\end{tabular}

La escala breve de estrés postraumático (PTSD) (Breslau et al., 1999), obtuvo una consistencia interna de $\mathrm{a}=.748$ con 7 elementos.
El $66 \%$ ha manifestado al menos un indicador de estrés postraumático con vivencia de violencia presencial y $22 \%$ de los participantes 
presentó frecuencia de síntomas de EPT, con dificultades para realizar actividades similares al contexto del evento vivido, con desmotivación, aislamiento, insomnio y aplanamiento afectivo según la Tabla 3. Sin diferencia significativa por sexo, la tendencia fue mayor en mujeres que en varones $(\mathrm{Mx}=1.97, \mathrm{Hx}=1.77)$.

\section{Correlaciones entre variables de estudio}

Se encontraron correlaciones positivas estadísticamente significativas entre violencia directa e indirecta con el ciberacoso $\left(\mathrm{r}=.213^{* *}\right.$; $\left.\mathrm{r}=.198^{* *}\right)$, este último correlacionó con violencia contextual en la escuela $\left(\mathrm{r}=.138^{*}\right)$, en la colonia $(\mathrm{r}=.122 *)$ y en los lugares de diversión $\left(\mathrm{r}=.181^{* *}\right)$. Asimismo, la violencia directa e indirecta con síntomas de estrés postraumático (ETP) $(\mathrm{r}=.394 * * ; \mathrm{r}=.177 * *)$. Este último con violencia presencial en general $(\mathrm{r}=.163 * *)$ y violencia contextual en la escuela $\left(\mathrm{r}=.125^{*}\right)$. Según la Tabla 4, los hallazgos señalan que la victimización contextual presencial correlacionó significativamente con el ciberacoso y la presencia de síntomas de estrés postraumático.

Tabla 3.

Síntomas de estrés postraumático

\begin{tabular}{llllll}
\hline & Sin síntoma & $\begin{array}{l}\text { Síntoma } \\
\text { bajo }\end{array}$ & $\begin{array}{l}\text { Síntoma } \\
\text { moderado }\end{array}$ & $\begin{array}{l}\text { Síntoma } \\
\text { alto }\end{array}$ & $\begin{array}{l}\text { Síntoma } \\
\text { significativo }\end{array}$ \\
\hline $\begin{array}{l}\text { Tiendo a recuperarme rápida- } \\
\text { mente después de haber pasado } \\
\text { tiempos difíciles }\end{array}$ & $20(7)$ & $40(13)$ & $68(23)$ & $95(31)$ & $77(26)$ \\
$\begin{array}{l}\text { Paso malos ratos tratando de } \\
\text { superar eventos estresantes }\end{array}$ & $37(12)$ & $65(22)$ & $95(32)$ & $81(27)$ & $22(8)$ \\
$\begin{array}{l}\text { Me toma largo tiempo el } \\
\text { recuperarme de eventos }\end{array}$ & $59(20)$ & $95(31)$ & $86(29)$ & $44(15)$ & $16(5)$ \\
$\begin{array}{l}\text { estresantes } \\
\text { Es difícil para mí recuperarme } \\
\text { cuando algo malo me pasa } \\
\begin{array}{l}\text { Usualmente supero los tiempos } \\
\text { difíciles con pocos problemas }\end{array}\end{array}$ & $56(19)$ & $89(30)$ & $83(28)$ & $53(18)$ & $19(6)$ \\
$\begin{array}{l}\text { Tiendo a tardar mucho para su- } \\
\text { perar los reveses en mi vida }\end{array}$ & $75(25)$ & $82(27)$ & $82(27)$ & $38(13)$ & $23(8)$ \\
\hline
\end{tabular}


Tabla 4

Correlaciones entre variables

\begin{tabular}{llllll}
\hline & Ciberacoso & $\begin{array}{l}\text { Estrés Pos- } \\
\text { traumático }\end{array}$ & $\begin{array}{l}\text { Violencia } \\
\text { en Colonia }\end{array}$ & $\begin{array}{l}\text { Violencia en } \\
\text { lugares de } \\
\text { diversión }\end{array}$ & $\begin{array}{l}\text { Violencia } \\
\text { en escuela }\end{array}$ \\
\hline $\begin{array}{l}\text { Violencia directa/ } \\
\text { presencial }\end{array}$ & $\begin{array}{l}.213^{* *} \\
(.000)\end{array}$ & $.394^{* *}(.000)$ & $\begin{array}{l}.526^{* *} \\
(.000)\end{array}$ & $.607^{* *}(.000)$ & $.701^{* *}$ \\
Violencia indirecta & $.198^{* *}$ & $.000)$ & & \\
Ciberacoso & $(.001)$ & $.177^{* *}(.002)$ & $\begin{array}{l}.276^{* *} \\
(.000)\end{array}$ & $.201^{* *}(.000)$ & $.176^{*}(.025)$ \\
$\begin{array}{l}\text { Estrés Pos- } \\
\text { traumático }\end{array}$ & & & $.122^{*}(.035)$ & $.181^{* *}(.002)$ & $.138^{*}(.017)$ \\
\hline
\end{tabular}

\section{Discusión}

La prevalencia de síntomas de estrés postraumático (TEPT) es relevante en la comunidad universitaria de Ciudad Juárez, México. El 22\% de la población encuestada reportó mantener al menos tres de los síntomas caracterizados en este padecimiento: dificultad con niveles importantes de angustia y ansiedad en el recuerdo de la experiencia vivida, insomnio, pesadillas, imágenes constantes e involuntarias relacionadas con el evento traumático, conductas de evitación de situaciones asociadas al hecho traumático, respuestas de hiperactivación con dificultad para concentrarse e irritabilidad, de acuerdo a la sintomatología establecida por la APA (2014). De acuerdo con lo reportado por Medina-Mora, et al. (2005) y por MendozaMojica et al (2013), la prevalencia de sucesos traumáticos asociados al desarrollo del TEPT es considerada de impacto en la integridad física y emocional de estudiantes universitarios.
Aunque ha sido clara la sintomatología del TEPT respecto a la reiteración de imágenes relacionadas con el evento traumático flashback-, el intento deliberado de su evitación, hiperactivación y embotamiento afectivo, es importante considerar estas experiencias fenomenológicas según el componente subjetivo y el contexto personal-social. Por lo ello, el horizonte clínico psicopatologizante con estudiantes universitarios de la zona norte de México tiende a variar; ubicando cierta discusión del concepto del TEPT, más que trastorno lineal y estático ubicado como trastorno de ansiedad en el anterior DSM-IV, lo hemos considerado como un estresor complejo con diversas formas de reacción de acuerdo con el nuevo DSM-V (Cova et al., 2011). Por 1o anterior, es preciso considerar el adecuado y no precoz diagnóstico del TEPT al estar presente en su inicio de forma parcial y agudizarse tras una circunstancia determinada presentando todos 
los síntomas necesarios para su presentación típica, de acuerdo con Ojeda-Vicente y GarcíaAbreu (2011).

Los efectos mostrados en síntomas de EPT no solo estuvieron relacionados con experiencias de violencia vivida sino también con experiencias de violencia social en contextos escolares, habitacionales y en lugares de diversión, así como en el ambiente del Internet. Los jóvenes universitarios mantienen comunicación activa mediante redes sociales electrónicas por diversos motivos: de diversión, académicos y/o laborales; no obstante, de existir un grado mínimo de ciberacoso, a comparación de otros niveles educativos previos, este estuvo relacionado significativamente con los síntomas de EPT, concordando con los hallazgos obtenidos por De la Rosa y Cárdenas (2012).

Las correlaciones bivariadas entre las variables del estudio indicaron que, en un contexto de violencia continua y permanente como el que atraviesa Ciudad Juárez, Chihuahua, México, se identifica en casi la cuarta parte de la población, al menos tres síntomas del EPT tipificados en el manual DSM-IV, que acompañan a los estudiantes universitarios en su cotidianidad. Aun estando presentes, una mínima parte se concientiza para atenderse psicológicamente, pues gran parte ha desarrollado factores resilientes de afrontamiento, sin la total desaparición de los síntomas EPT. Es pertinente entonces, generar programas públicos y privados de atención que permitan abordar de manera integral, interdisciplinaria y sistémica, estos síntomas EPT en beneficio de la calidad de vida de los ciudadanos de una comunidad en permanente violencia contextual.

\section{Referencias}

Abramovay, M. (2005). Debate: violência, mediação e convivência na escola. Recuperado de: http://cdnbi.tvescola.org. br/resources/VMSResources/contents/ document/publicationsSeries/215810Debate violencia.pdf

Alvarado, A. (2013). La violencia juvenil en América Latina. Estudios Sociológicos, 31(91), 229-258. Recuperado de: http://www. redalyc.org/articulo.oa?id=59830136009

Andrade-Salazar, J. (2008). Efectos psicopatológicos del conflicto armado colombiano en familias en situación de desplazamiento forzado reasentadas en el municipio del Cairo en el año 2008. Orbis. Revista Cientifica Ciencias Humanas, (7)20, 111-114.

Asociación Americana de Psiquiatría (1995). Manual diagnóstico y estadístico de los trastornos mentales (DSM-IV). 4ta edición. Washington, EUA: Masson.

Asociación Americana de Psiquiatría (2014). Manual diagnóstico y estadístico de los trastornos mentales (DSM-5). 5ta. edición. Arlington, EUA: Asociación Americana de Psiquiatría.

Arteaga-Botello, N. (2003). El espacio de la violencia: un modelo de interpretación social. Sociológica, (18)53, 119-145. 
Breslau, N., Peterson, E., Kessler, R., \& Shultz, L. (1999). Short screening Scale for DSMIV Posttraumatic Stress Disorder. American Journal of Psychiatry, (156)6, 908-911.

Briceño-León, R. (2008). La violencia homicida en América Latina. América Latina Hoy, (50), 103-116.

Buelga, S., Cava, M., \& Musitu, G. (2010). Cyberbullying: victimización entre adolescentes a través del teléfono móvil y de Internet. Psicothema, (22)4, 784-789.

Carvajal, C. (2002). Trastorno por estrés postraumático: aspectos clínicos. Revista chilena de neuro-psiquiatría, 40(2), 20$34 . \quad \mathrm{https}: / / \mathrm{dx}$.doi.org/10.4067/S071792272002000600003

Chávez, M., Petrzelová, J., Zapata, J., Hernández, M., \& Rodríguez, M. (2015). Violencia psicosocial en el noreste de México: repercusiones en la salud mental. Psicogente, 18(33), 11-21. http://doi.org/10.17081/ psico.18.33.52.

Cova, F., Rincón, P., Grandón, P., \& Vicente, B. (2011). Controversias respecto de la conceptualización del trastorno de estrés postraumático. Revista Chilena NeuroPsiquiatría, 49(3), 288-297

De la Rosa, A., \& Cárdenas, G. (2012). Trastorno por estrés postraumático: eficacia de un programa de tratamiento mediante realidad virtual para víctimas de violencia criminal en población mexicana. Anuario de Psicología, 42(3), 377-391. Recuperado de: http://www. redalyc.org/articulo.oa?id $=97026839008$
Echeburúa, E., Corral, P., Amor, P., Zubizarreta, I., \& Sarasua, B. (1997). Escala de gravedad de síntomas del trastorno de estrés postraumático: propiedades psicométricas. Análisis y Modificación de Conducta, (23)90, 503-526.

BM Banco Mundial (2012). La violencia juvenil en México. Reporte de la situación, el marco legal y los programas gubernamentales. Recuperado de: www.defensoria.ipn.mx/.../ Estudio-Violencia-juvenil

Emir, A. (2015). Ciberbullying, cuando el acoso sale de las escuelas. México: La Jornada. Recuperado de: http://www.jornada.unam. $\mathrm{mx} / 2015 / 01 / 26 /$ sociedad/037n1 soc

Estévez, A., Villardón, L., Calvete, E. Padilla, P., \& Orue, I. (2010). Adolescentes víctimas de cyberbullying: Prevalencia y características. Behavioral Psychology/Psicología Conductual, (18)1, 73-89.

García-Maldonado, G., Martínez-Salazar, G., Saldívar-González, A., Sánchez-Nuncio, R., Martínez-Peralez, G., \& BarrientosGómez, M. (2012). Factores de riesgo y consecuencias del cyberbullying en un grupo de adolescentes. Boletín médico del Hospital Infantil de México, 69(6), 463-474. Recuperado de: http:/www.scielo.org.mx/ scielo.php?script $=$ sci_arttext\&pid $=\mathrm{S} 1665$ $11462012000600007 \& \operatorname{lng}=\mathrm{es} \& \operatorname{tlng}=\mathrm{es}$.

González-Valles, M. N., \& Romero-Cruz, B. (2015). Violencia masiva y sensibilidad social infantil. En Castro-Valles, A. (Coord.). Ejercicio de la violencia en Ciudad Juárez, 
Chihuahua (1-19). Ciudad Juárez, México: Universidad Autónoma de Ciudad Juárez.

Gurrola-Peña, G., Balcazar-Nava, P., \& Moysen, A. Cuestionario denominado Escala de Exposición de la Violencia [EVV]. Toluca: Universidad Autónoma del Estado de México. (Inédito).

Herrera-López, V., \& Cruzado, L. (2014). Estrés postraumático y comorbilidad asociada en víctimas de la violencia política de una comunidad campesina de Huancavelica, Perú. Revista de Neuropsiquiatría, 77(3), 144-159.

Jaitman, L. (2015). Los costos del crimen y la violencia en el bienestar en América Latina $y$ el Caribe. Nueva York, EUA: Banco Interamericano de Desarrollo.

Krug, E., Mercy, J., Dahlberg, L., \& Zwi, A. (2002). El informe mundial sobre la violencia y la salud. Biomédica, 22(2), 327-336.

Laca-Arocena, F., \& Navarro-Camarena, F. (2013). La percepción de la violencia en México en relación con el bienestar subjetivo y social. Anuario de Psicología, 43(3) 323334. Recuperado de: http://www.redalyc.org/ articulo.oa?id=97030658004.

Leiner, M., Villanos, M., Puertas, H., Peinado, J., Ávila, C., \& Dwivedi, A. (2015). The emotional and behavioral problems of children exposed to poverty and/or collective violence in communities at the MexicoUnited States border: A comparative study. Salud Mental, 38(2), 95-102.
Medina-Mora, M. E, Borges-Guimaraes, G., Lara, C., Ramos-Lira, L., Zambrano, J., \& Fleiz-Bautista, C. (2005). Prevalencia de sucesos violentos y de trastorno por estrés postraumático en la población mexicana. Salud Pública de México, 47(1), 8-21. Recuperado de: http://www.scielo.org.mx/ scielo.php?script=sci_arttext\&pid=S003636342005000100004\&lng=es\&tlng=es

Mendoza-Mojica, S.A, Márquez-Mendoza, O., Guadarrama-Guadarrama, R., \& RamosLira, R.E. (2013). Medición del Trastorno por Estrés Postraumático (TEPT) en universitarios mexicanos. Salud Mental, 36, 493-503.

Médicos Sin Fronteras. (2010). Tres veces víctimas. Víctimas de la violencia, el silencio $y$ el abandono. Conflicto armado y salud mental en el departamento de Caquetá, Colombia. Barcelona, España: Médicos Sin Fronteras. Recuperado de: https://www. msf.es/sites/default/files/adjuntos/InformeCOLOMBIA-2010.pdf.

Médicos Sin Fronteras. (2013). Las heridas menos visibles: Salud mental, violencia y conflicto armado en el sur de Colombia. Barcelona, España: Médicos Sin Fronteras. Recuperado de: https://www.msf.es/sites/ default/files/adjuntos/Informe-Colombia_ Junio-2013.pdf

Observatorio Nacional Ciudadano. (2015). Incidencia de los delitos de alto impacto en México. Reporte anual. Recuperado de: http:// 
onc.org.mx/wp-content/uploads/2016/06/ REPORTE-ANUAL-2015.pdf

Ojeda-Vicente, M., \& García-Abreu, D. (2011).

El estrés postraumático, un diagnóstico a tener en cuenta. Revista Cubana de Medicina Militar, 40(2), 176-181.

Organización Mundial de la Salud. (2002). Informe mundial sobre la violencia y la salud. Resumen. Washington, EUA: OPS. Recuperado de: http://www.who.int/ violence_injury_prevention/violence/world_ report/es/summary_es.pdf

Prieto-Quezada, M., Carrillo-Navarro, J., \& Lucio-López, L. (2015) Violencia virtual y acoso escolar entre estudiantes universitarios: el lado oscuro de las redes sociales. Innovación Educativa, (15)68, 33

Rodríguez Ferreira, A. (2016). Violent Mexico: Participatory and multipolar violence associated with organised crime. International Journal of Conflict and Violence, 10(1), 4060.

Ruíz, J.I. (2007). Cultura ciudadana, miedo al crimen y victimización: un análisis de sus interrelaciones desde la perspectiva del tejido social. Acta Colombiana de Psicología, 10(1), 65-74.

Serrano Barquín, C., \& Serrano Barquín, R. (2014). Ciberacoso en estudiantes universitarios: diagnóstico y medidas correctivas para intervenir en la violencia de género expresada en redes sociales. Revista de Comunicación de la SEECI, Número extraordinario, 94-101.
Sociedad Mexicana de Psicología. (2009). Código ético del psicólogo. México: SMP. Vega-López, M., González-Pérez, G., \& Quintero-Vega, P. (2013). Cyberbullying: Victimization of Students of Public Secondary School in Tlaquepaque, Jalisco, Mexico. Revista de Educación y Desarrollo, 25, 13-20.

Wieviorka, M. (1997). O novo paradigma da violência. Tempo Social, 9(1), 5-41. Recuperado de: http:/www.scielo.br/scielo.php?script $=$ sci_arttext $\& p i d=S 0103-20701997000100002 \&$ lng=pt\&nrm=iso\&tlng=en

Recibido: 06/06/2019

Revisado: 24/06/2019

Aceptado: 29/11/2019 